\title{
Estimação do Conteúdo Eletrônico Total da Ionosfera utilizando Geoestatística.
}

\author{
Total Electronic Content Estimation using Geostatistics.
}

\author{
Everson Mattos , Adriano Petry , Denilson Kulman, Franciele dos Santos Padilha \\ e Guilherme Hollweg \\ Instituto Nacional de Pesquisas Espaciais - INPE- Brasil \\ everson.mattos@inpe.br*,dr.adrino.petry@gmail.com,denilsonkulman@hotmail.com, franceckhardt@gmail.com, \\ guilhermehollweg@hotmail.com
}

\begin{abstract}
Resumo
A ionosfera é a camada ionizável da atmosfera, onde ocorrem processos físicos e químicos que resultam na ionização e neutralização de átomos, que interagem com o campo magnético terrestre e geram regiões com maior ou menor concentração eletrônica. A distribuição de ionização presente na ionosfera afeta principalmente as telecomunicações, podendo causar distorções e falhas em sistemas como o Sistema Global de Posicionamento (GPS). Neste trabalho, são utilizados dados de Conteúdo Eletrônico Total (TEC) observados através de estações de GPS de dupla frequência, espalhadas na América do Sul, para estimação do TEC em toda a região, incluindo as localidades que não dispõem dessas medições. Para isso, foram aplicadas as técnicas geostatísticas krigagem ordinária e simulação sequencial gaussiana através da ferramenta de software Stanford Geostatistical Modeling Software (SGEMS), que apresentaram resultados similares e coerentes.
\end{abstract}

Palavras-chave: Dados de GPS. Krigagem Ordinária. Simulação Sequencial Gaussiana. Geoestatística

\begin{abstract}
Ionosphere is the ionizable atmosphere layer, where physical and chemical processes result in atoms ionization and neutralization, which interact with Earth's magnetic field and generate regions with different electronic concentration. The ionization distribution in ionosphere affects mainly telecommunications, and may cause distortions and systems failure such as Global Positioning System (GPS). In this work, Total Electronic Content (TEC) data is used, observed at several double frequency GPS stations over South America, to estimate TEC in the whole region, including locations where these measures are not available. The geostatistic techniques ordinary kriging and gaussian sequential simulation were applied, using the software Stanford Geostatistical Modeling Software (SGEMS), and presented similar and coherent results.
\end{abstract}

Keywords: GPS data. Ordinary Krigging. Gaussian Sequential Simulation. Geostatistic 


\section{Introdução}

Os fenômenos ionosféricos, assim como os fenômenos atmosféricos, possuem natureza caótica. Tais fenômenos são de difícil previsibilidade, e seu estudo requer grande quantidade de observações, obtidas através de diferentes instrumentos de medição (Petry et al., 2013). Estações de GPS de dupla frequência são capazes de estimar o TEC ao longo do caminho que liga a estação e cada satélite da constelação utilizado. A partir desses dados, é possível estimar o TEC vertical nas localidades próximas à estação.

Quando não se dispõe da cobertura instrumental ideal, uma alternativa para preencher as lacunas existentes nos dados é utilizar algum método de estimação. A interpolação é um desses métodos e pode ser dividida em dois grupos: não estatísticos e estatísticos. Dentro da família dos interpoladores estatísticos, os interpoladores do tipo Best Linear Unbased Estimator(BLUE), permitem verificar a variância do erro de estimação, e assim minimizá-la. Com isso, representam mais fielmente os dados, quando comparados com outros métodos de interpolação. Esses interpoladores são considerados ótimos. Porém, a prática mostra que para grande quantidade de pontos analisados, a complexidade computacional aumenta consideravelmente.

Neste trabalho, foram usados métodos estatísticos, pois permitem, além de estimar valores em localidades ausentes de observações, avaliar o erro cometido nas interpolações. A estatística aplicada, em especial a geoestatística, usa modelos matemáticos para representar fenômenos naturais, que variam suas propriedades em função da localização espacial (Simoes et al., 2007).

A geoestatística pressupõe correlação e continuidade espaciais entre amostras. Assim, quanto mais próximas as amostras estiverem, mais relacionadas estarão, tendendo assim a serem mais semelhantes que amostras mais distantes. Ou seja, próximo a um valor elevado, é bem provável que haja outro valor elevado (Andriotti, 2003).

\section{Metodologia}

Neste trabalho, foram utilizados dados oriundos de estações GPS do Instituto Brasileiro de Geografia e Estatística (IBGE) e da Low-Latitude Ionospheric Sensor Network (LISN). Os valores de TEC utilizados foram calculados utilizando o código desenvolvido na Universidade de Nagoya (Otsuka et al., 2002). As medidas obtidas fornecem os valores para número total de elétrons livres acumulados em altitude e formam mapas bi-dimensionais. O estudo foi realizado para o dia 19 de Março de 2011, nos horários 0h, 6h, 12h e 18h, conforme mostra a figura 1 .
A área de estudo compreendeu as coordenadas $95^{\circ} S$; $60^{\circ} \mathrm{W}$ e $26^{\circ} \mathrm{N} ; 30^{\circ} \mathrm{W}$, DATUM WGS 84 . Os dados para cada hora tratada foram avaliados de forma independente. A partir do variograma experimental dos dados, foram gerados e ajustados, de forma empírica, o variograma teórico para os modelo gaussiano, considerando as direções $\left(0^{\circ} ; 0^{\circ}\right)$ e $\left(90^{\circ} ; 0^{\circ}\right)$. A opção pelo modelo gaussiano se deu pela melhor conformidade com o variograma experimental, quando comparado aos modelos esférico e exponencial. A figura 2 mostra os variogramas experimental e teórico para as direções consideradas para a $0 \mathrm{~h}$. Em todos os casos, a variância a priori dos dados experimentais foi utilizada para estabelecer o patamar do variograma teórico.

Na sequência utilizou-se a krigagem ordinária e a simulação sequencial gaussiana para estimação dos dados desconhecidos. Após, foram analisados os dados da krigagem e sua variância, bem como a média e variância das simulações. A ferramenta de software SGEMS foi utilizada nas etapas descritas.

\section{Krigagem Ordinária}

A krigagem ordinária é uma generalização da krigagem simples, pois não requer o conhecimento da média do universo amostral e tampouco que essa média seja constante. Segundo Yamamotto e Landim (2013), dos métodos de krigagem é o mais utilizado. Por ser o método local de estimativa, o valor interpolado resulta da combinação linear das amostras vizinhas. A equação 1 mostra matematicamente essa técnica.

$$
\hat{Z}_{K O}\left(x_{o}\right)=\sum_{i=1}^{n} \lambda_{i} Z\left(x_{i}\right)
$$

onde:

$\hat{Z}_{K O}\left(x_{0}\right)$ é o valor da krigagem no ponto $x_{0}$;

$\lambda_{i}$ são os pesos do interpolador;

$Z\left(x_{i}\right)$ são os valores da variável aleatória.

Os pesos são calculados sob duas restrições. A primeira é que o estimador não seja enviesado, e a segunda que a variância da estimativa seja mínima. E a variância da estimativa é calculada de acordo com a equação 2:

$$
\sigma_{R}^{2}=\operatorname{Var}\left[Z\left(x_{0}\right)-\hat{Z}_{K O}\left(x_{0}\right)\right]
$$

\section{Simulação Sequencial Gaussiana}

Um dos problemas da interpolação por krigagem é que essa apresenta suavização, $R(x)$, ou seja, diminuição da amplitude de valores altos e aumento de valores 


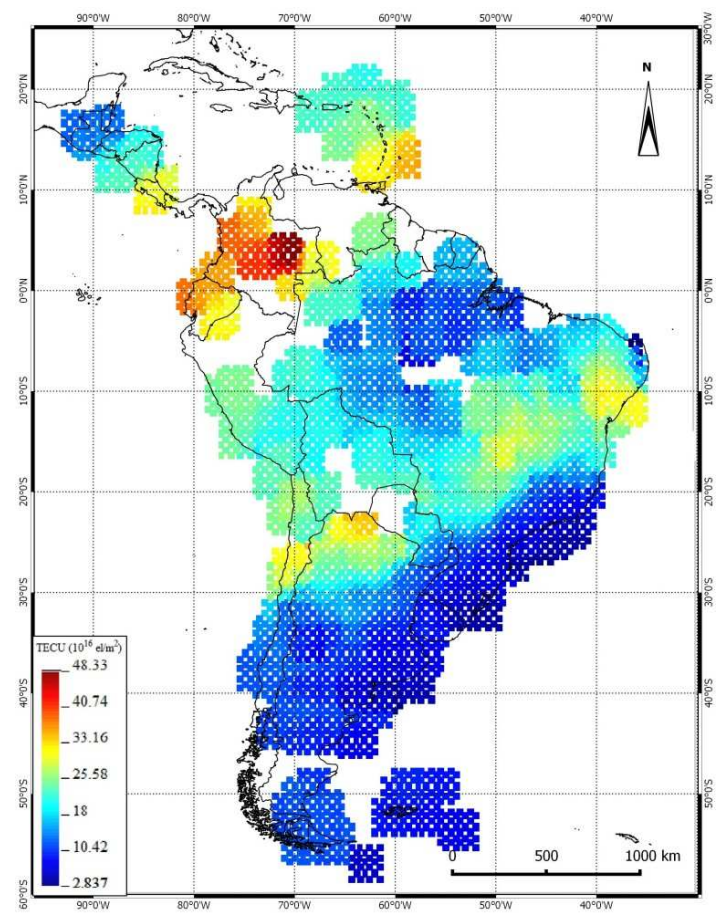

(a) $0 \mathrm{~h}$

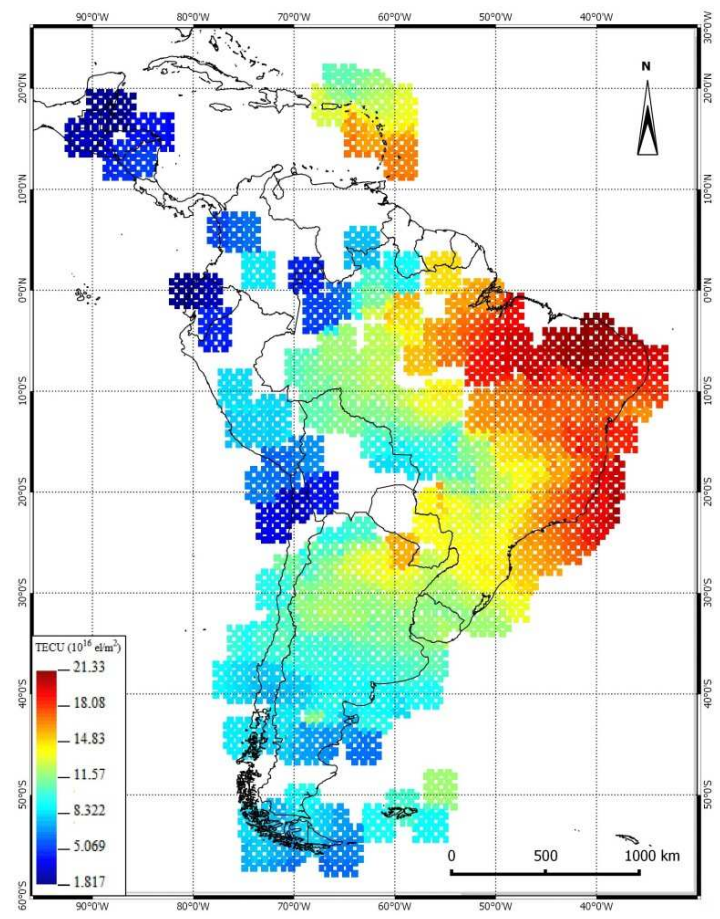

(c) $12 \mathrm{~h}$

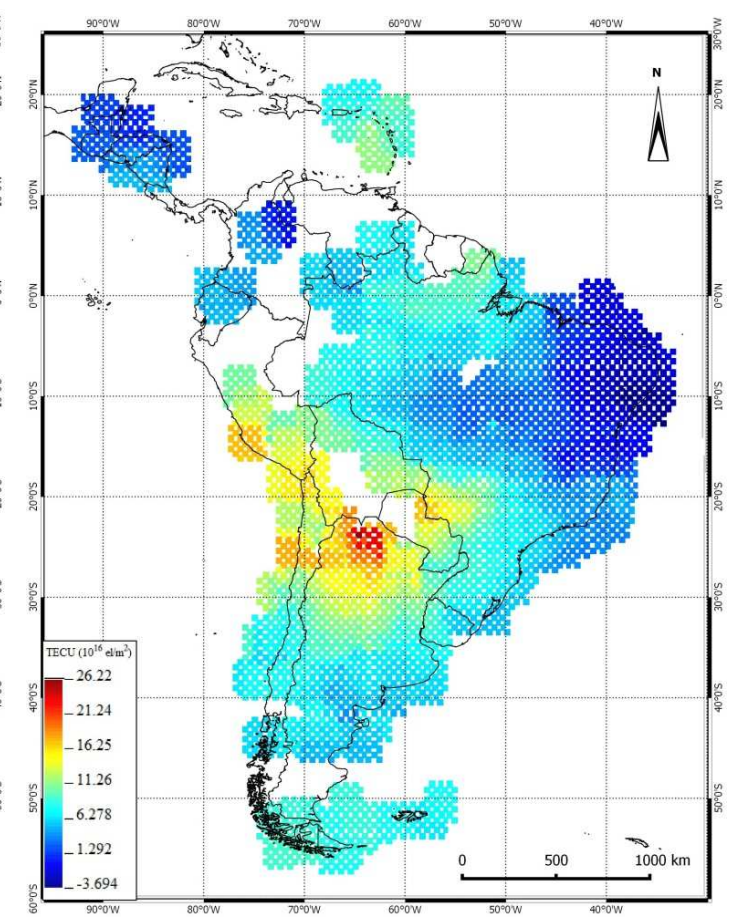

(b) $6 \mathrm{~h}$

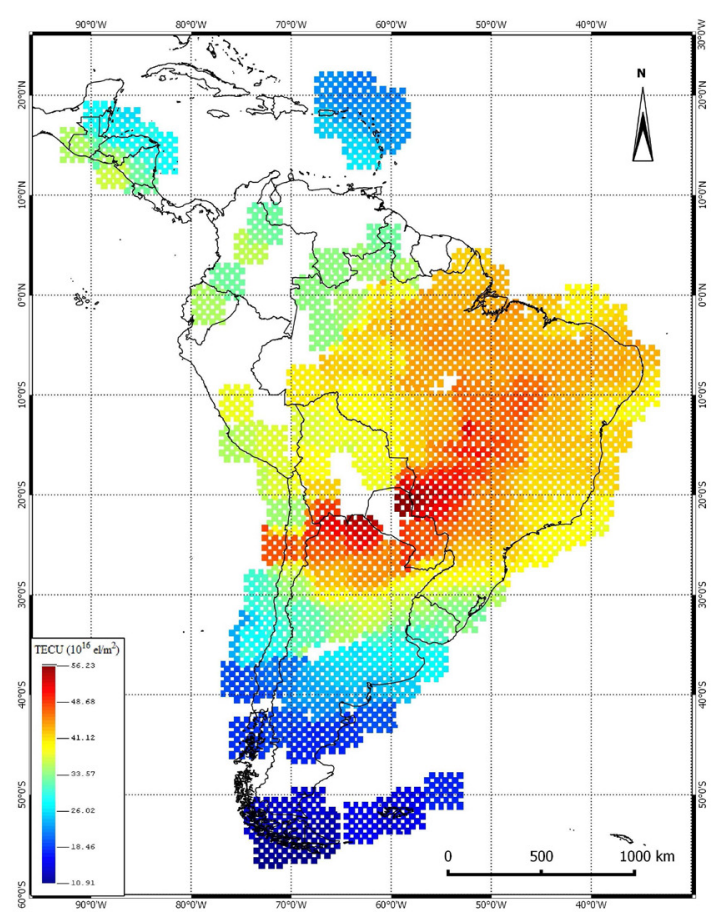

(d) $18 \mathrm{~h}$

Figura 1: Valores observados para TEC em 19 de março de 2011 


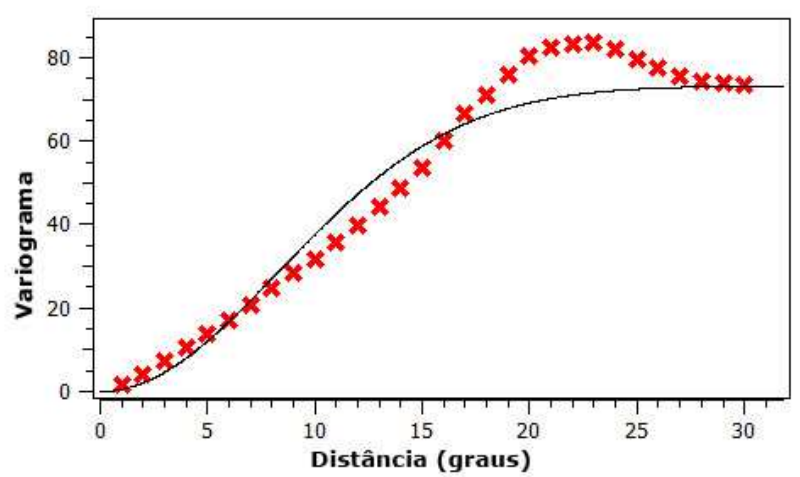

(a) Direção $(0,0)$

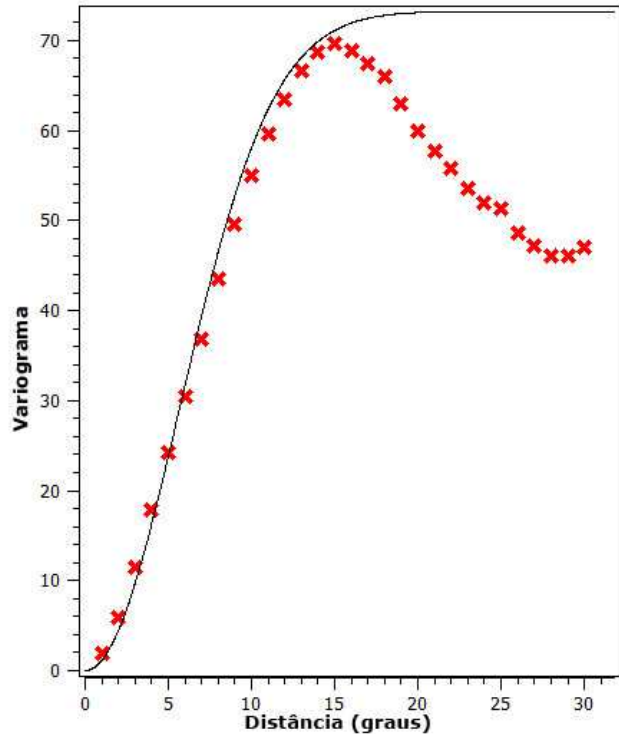

(b) Direção $(90,0)$

Figura 2: Ajuste do variograma para o modelo Gaussiano para 0h

baixos. A equação 3 mostra esse erro de suavização. Para resolver esse problema foram desenvolvidos os métodos de simulação estatística (Yamamotto e Landim, 2013).

$$
Z\left(x_{0}\right)=\hat{Z}_{K O}+R\left(x_{0}\right)
$$

Sendo $R\left(x_{0}\right)$ uma variável aleatória correspondente ao erro de estimação por suavização.

A simulação sequencial gaussiana é um processo de simulação ou estimação sobre os nós de uma malha, onde cada ponto estimado está condicionado aos demais estimados anteriormente. A implantação do princípio de simulação sequencial, sob a suposição de uma função aleatória multigaussiana, define a chamada simulação gaussiana sequencial.

Os métodos de simulação geram soluções equiprováveis para o erro de estimação conforme equação 4, respeitando o histograma dos dados e o modelo usado para ajuste do variograma. A isso chama-se de precisão global, o que não ocorre com a krigagem, que possui precisão local, traduzida pela alta correlação entre os pontos estimados e as amostras.

$$
\begin{gathered}
F_{N}\left(x_{1}, \ldots, X_{N} ; z_{1}, \ldots, z_{N} \mid(n)\right)= \\
F\left(x_{1} ; z_{1} \mid(n)\right) \ldots \\
F\left(x_{N} ; z_{N} \mid(n+N+1)\right)
\end{gathered}
$$

A equação 4 é a função teórica do método de simulação estatística gaussiana. Cada novo ponto simulado é usado para atualizar a função de distribuição acumulada condicional, que gera os pontos simulados fazendo uso do método de Monte Carlo.

\section{Resultados}

Após a inserção dos dados observacionais, análise e definição do variograma teórico, a krigagem ordinária foi aplicada aos dados, resultando nos mapas mostrados na figura 3.

Como pode ser visto a krigagem produz suavização nos pontos de alta concentração eletrônica e tende a aumentar os valores de baixa concentração, funcionando como uma espécie de filtro passa-baixas, como o de média móveis. Salienta-se que a ferramenta SGEMS requer a definição das dimensões de um elipsóide para limitar os dados a serem usados em cada interpolação. Isso resultou em um mapa incompleto, onde pontos demasiadamente afastados de dados observacionais não foram estimados. Pode-se, contudo, reduzir a área não interpolada aumentando-se as dimensões do elipsóide.

Uma limitação observada na ferramenta de software utilizada foi que o variograma teórico, empiricamente definido, era necessariamente o mesmo para todas as direções avaliadas. Assim, caso os variogramas experimentais sejam muito diferentes, dificilmente alcançaremos um variograma experimental adequado para as duas direções avaliadas.

Para a simulação sequencial gaussiana, os mapas de resultado médio para 400 simulações para cada hora são mostrados na figura 4 .

Ao comparar os resultados obtidos com a krigagem ordinária, percebemos uma boa concordância entre os resultados. A simulação também fornece uma estimativa de erro para as estimações, traduzido pelos mapas de variância mostrados na figura 5 . 


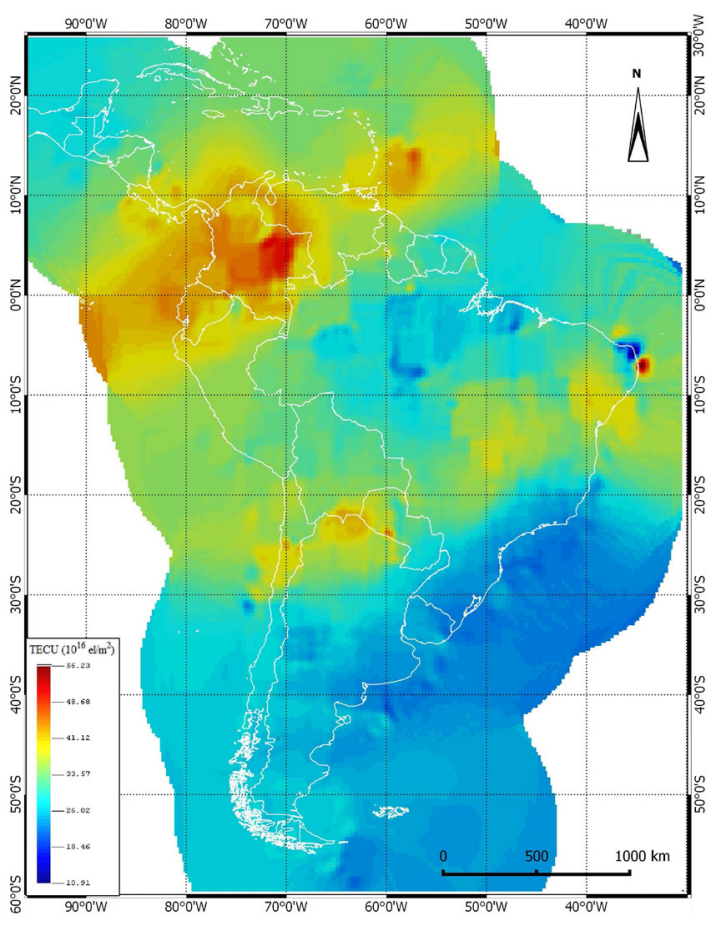

(a) $0 \mathrm{~h}$

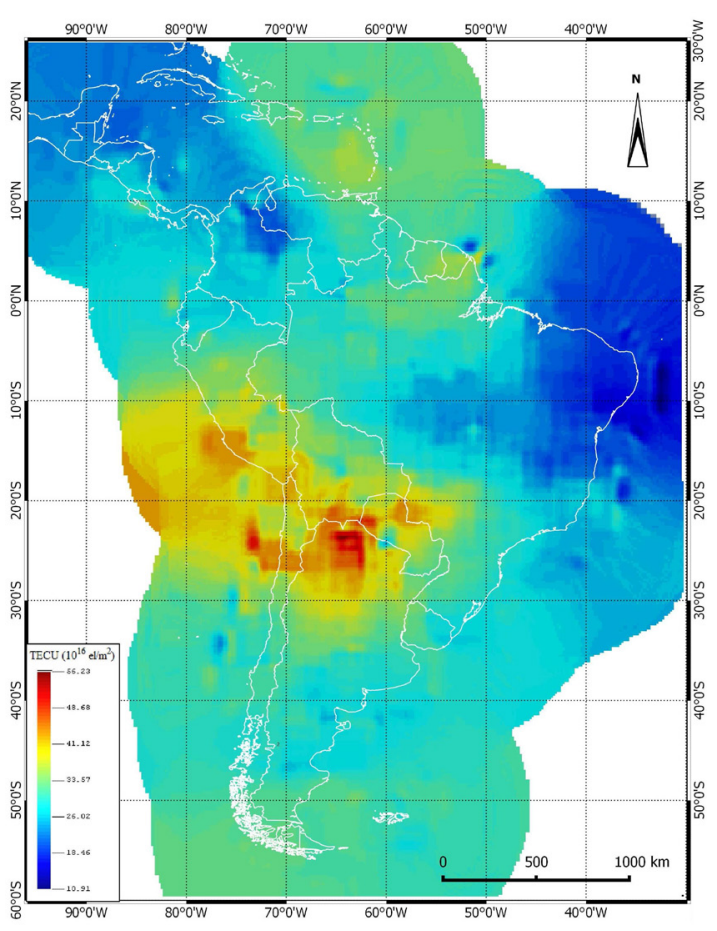

(b) $6 \mathrm{~h}$

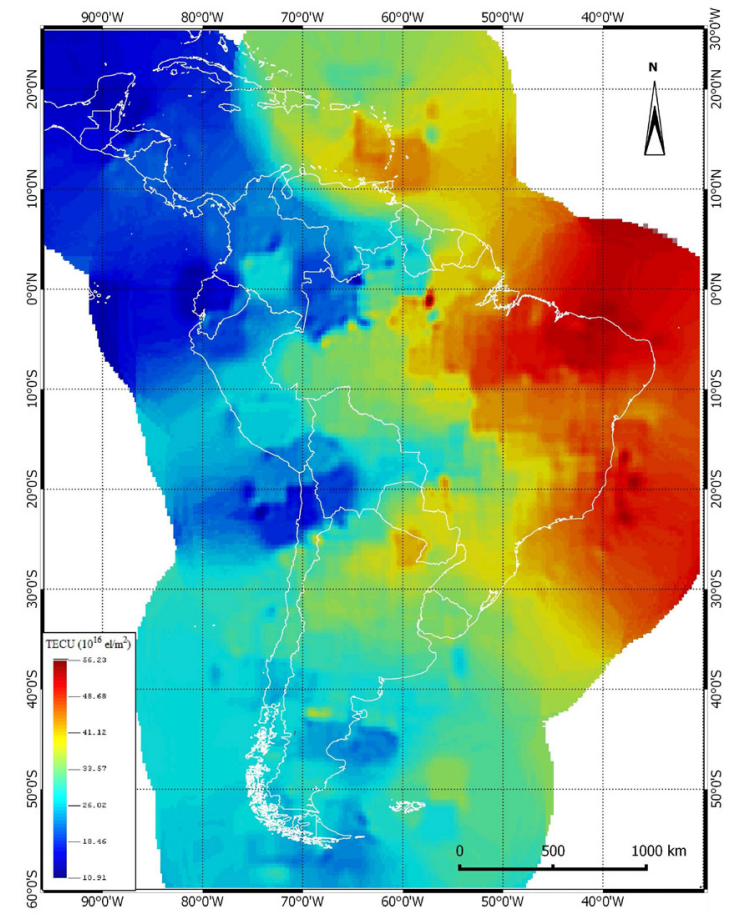

(c) $12 \mathrm{~h}$

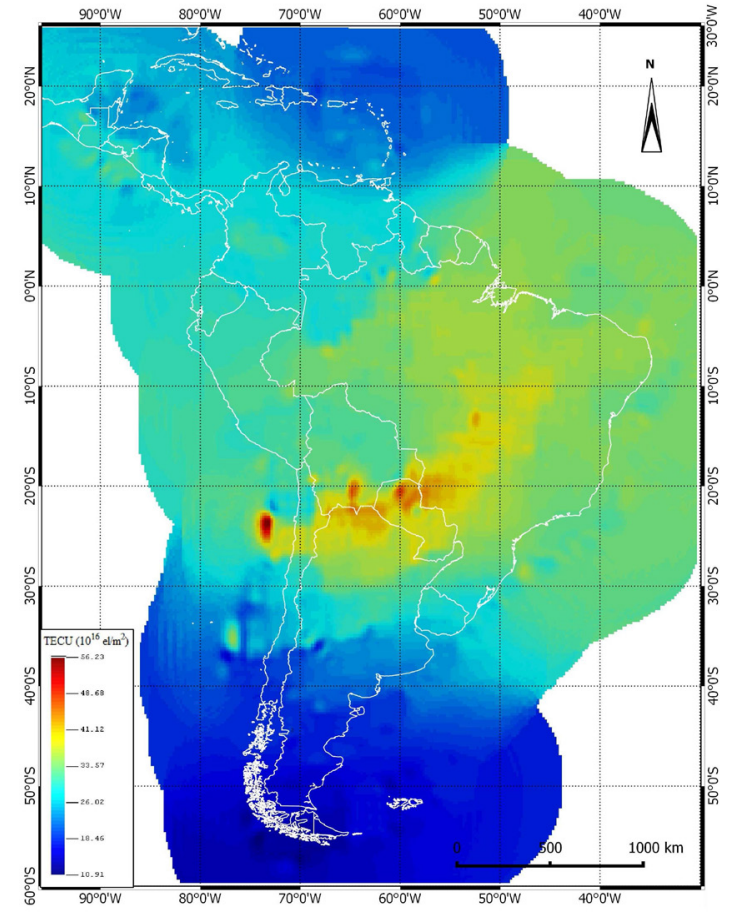

(d) $18 \mathrm{~h}$

Figura 3: Resultado da Krigagem 


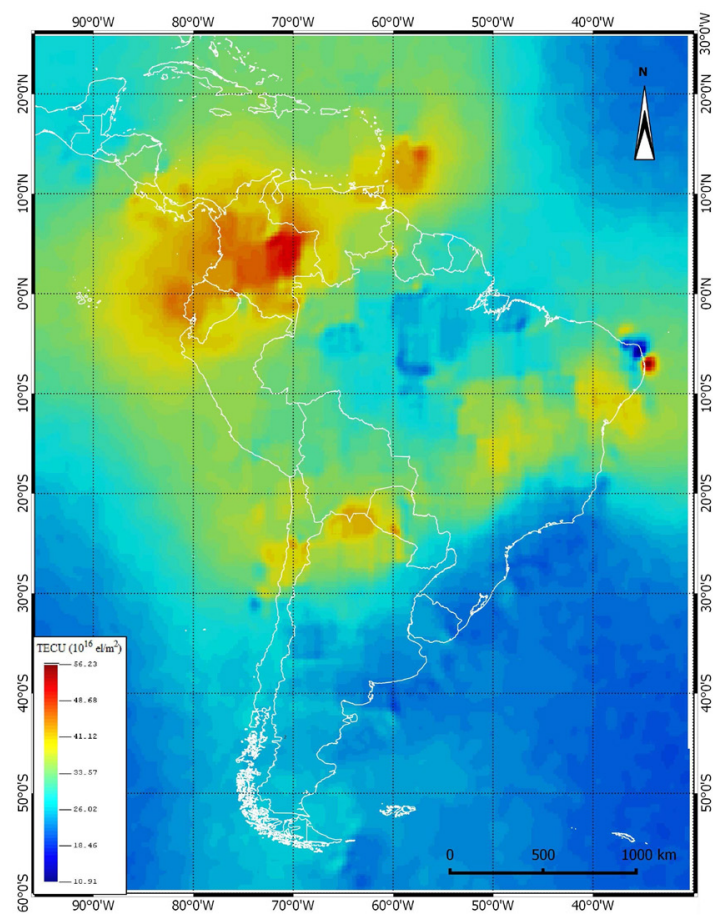

(a) $0 \mathrm{~h}$

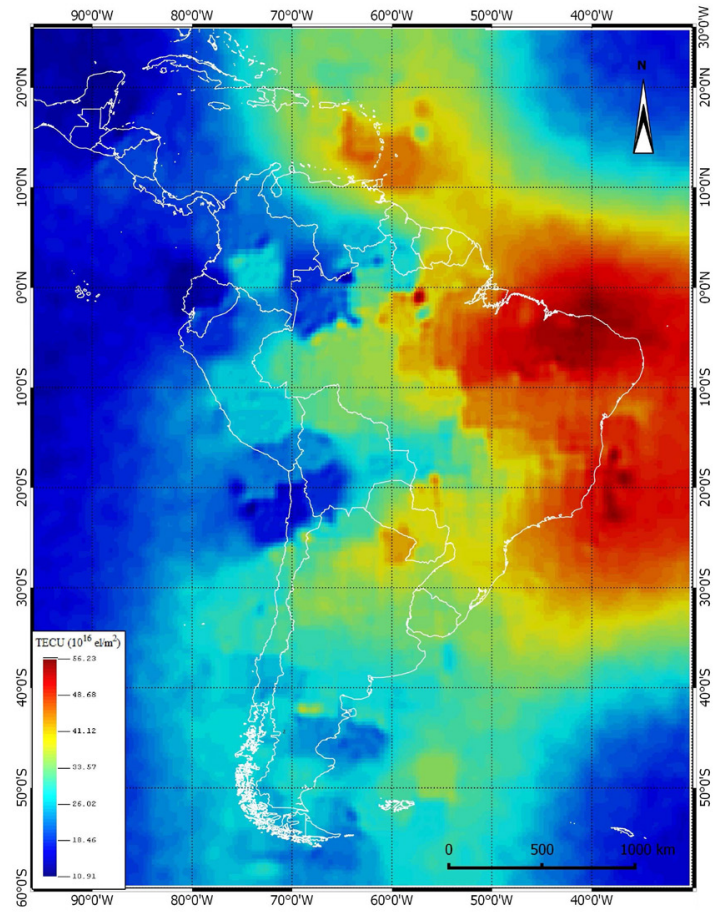

(c) $12 \mathrm{~h}$

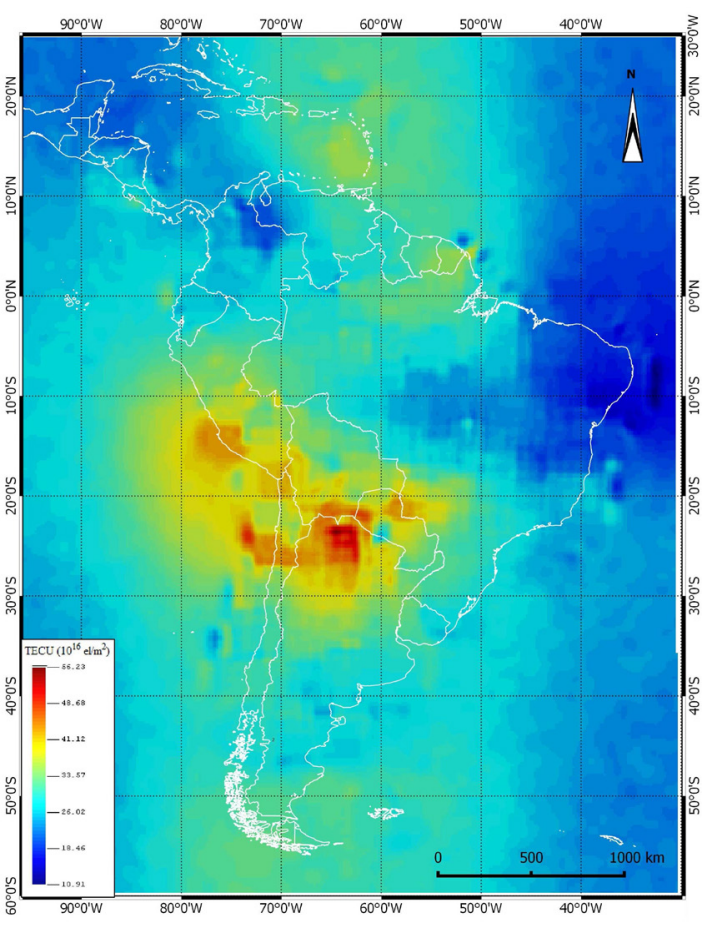

(b) $6 \mathrm{~h}$

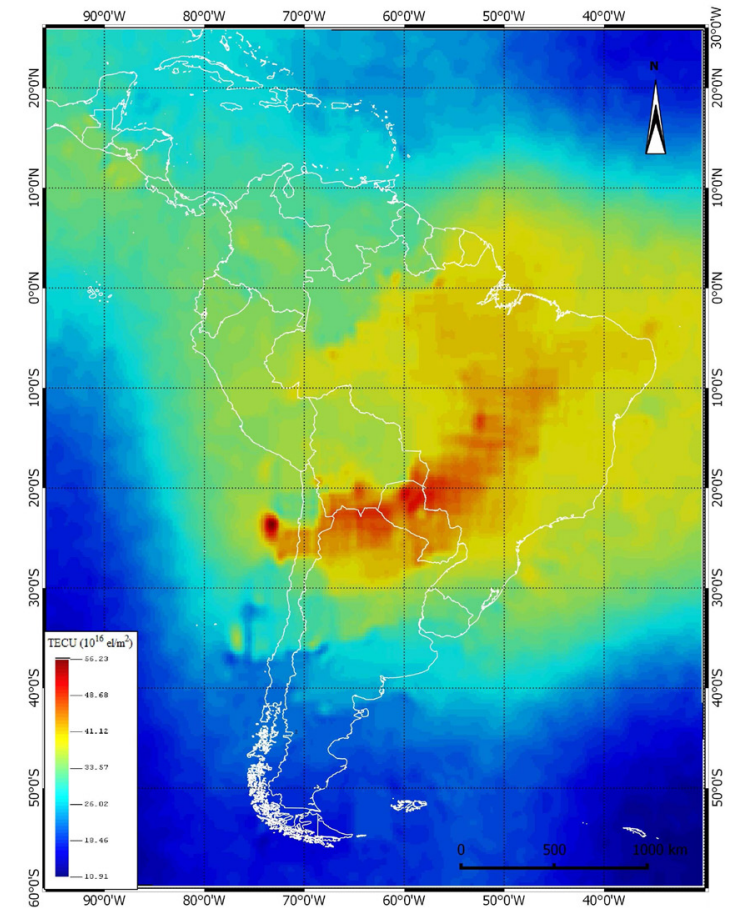

(d) $18 \mathrm{~h}$

Figura 4: Resultado médio para Simulação Sequencial Gaussiana 


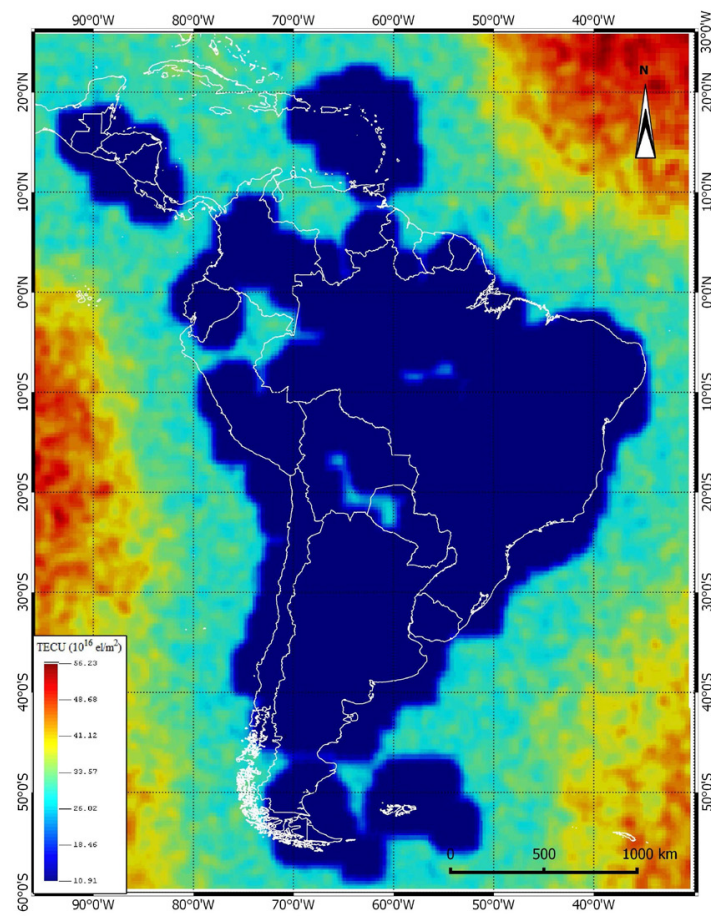

(a) $0 \mathrm{~h}$

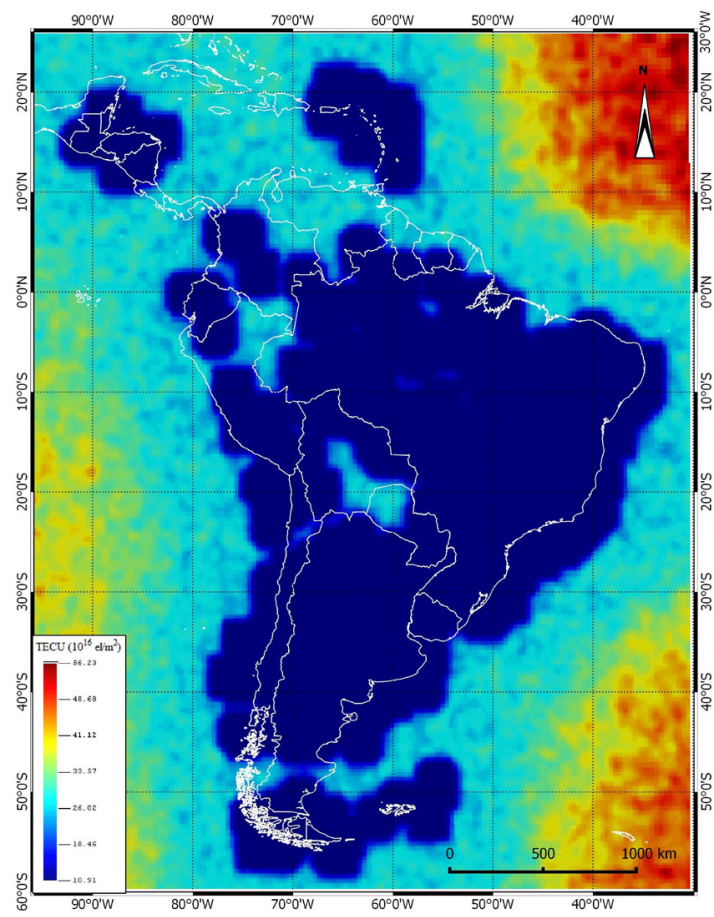

(c) $12 \mathrm{~h}$

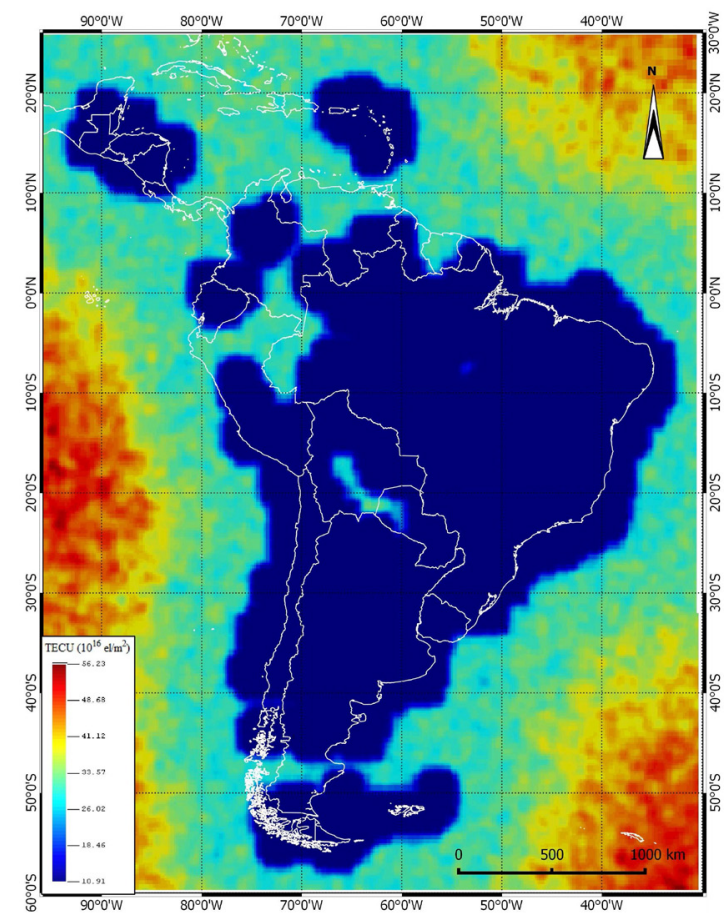

(b) $6 \mathrm{~h}$

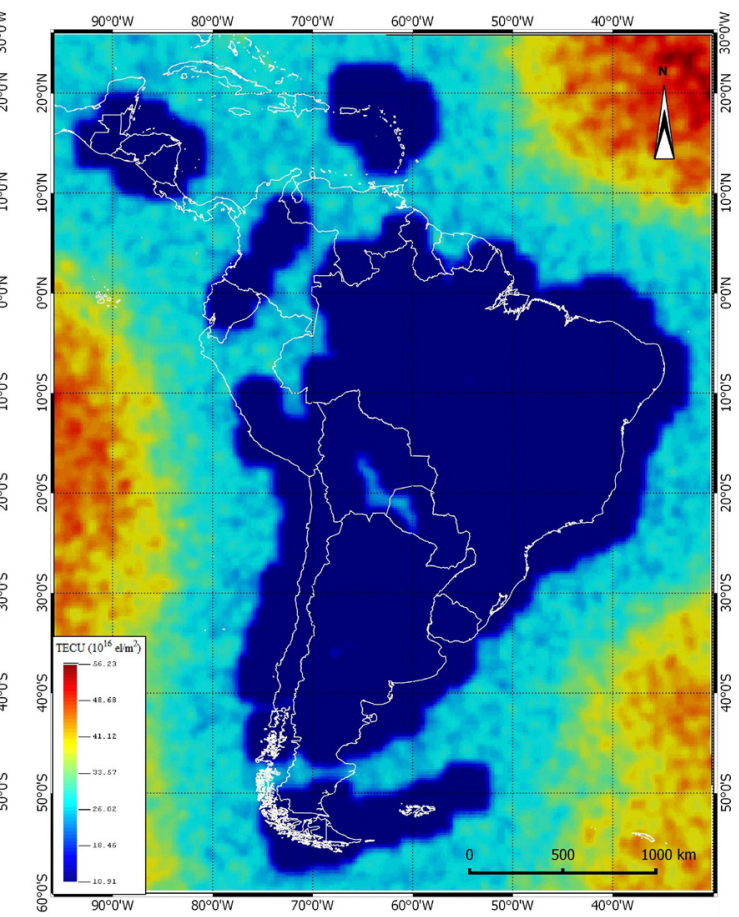

(d) $18 \mathrm{~h}$

Figura 5: Variância para Simulação Sequencial Gaussiana 
Como esperado, a confiança nas estimativas próximas a dados de observação foi maior, quando comparadas às estimativas mais afastadas, que apresentaram valores de variância bem mais elevados. O tempo para processamento dos dados da simulação foi consideravelmente superior ao necessário para realizar a krigagem. Quanto maior os número de simulações utilizado, maior o tempo requerido.

\section{Conclusões}

Dados de TEC observados através de estações de GPS na América do Sul foram utilizados para estimação de valores em regiões não cobertas pelas observações. Uma vez que a dinâmica ionosférica varia significativamente em uma mesma região em horários distintos, em razão principalmente da ionização gerada pela atividade solar, as interpolações foram realizadas independentemente em 4 horários distintos $(0 \mathrm{~h}, 6 \mathrm{~h}, 12 \mathrm{~h}$, e 18h) para o dia 11 de março de 2011. Os resultados obtidos com a krigagem ordinária foram semelhantes aos obtidos com a simulação sequencial gaussiana em todos os horários estudados, e mostraram-se coerentes com o que poderia se esperar, considerando a distribuição de TEC disponível através das observações.

A variância da simulação geoestatística forneceu, também, uma estimativa de confiança para os valores médios interpolados. Como esperado, as regiões mais afastadas das localidades de observações apresentaram variâncias superiores. A ferramenta de software utilizada (SGEMS) apresentou algumas limitações em termos de definição de variogramas teóricos a partir dos dados experimentais, falta de clareza no entendimento e definição de parâmetros empíricos como dimensões do elipsóide utilizado na krigagem, e vários problemas com travamentos e demora excessiva para conclusão dos processamentos. Por outro lado, a fácil visualização dos dados e resultados facilitaram o entendimento e funcionamento do software.

\section{Agradecimentos}

\section{Referências}

Andriotti, J., 2003. Fundamentos de estatística e geoestatística. Editora UNISINOS.

Otsuka, Y., T.Ogawa, Saito, A., Tsugawa, T., Fukao, S., Miyazaki, S., 2002. A new technique for mapping of total electron content using gps network in japan. Earth Planets Space 54, 63-70.

Petry, A., Mattos, E., Leopoldino, T. M., de Souza, J. R., August 26-29, 2013. 2013. Ionospheric 3d-grid inter- polation for the brazilian ionosphere dynamics forecasting system. Brazilian Geophysical Society 31 (7), 799-815, thirteenth International Congress of The Brazilian Geophysical Society.

Simoes, M., Meirelles, P., Camara, G., Almeida, C. M., 2007. Geomatica Modelos e Aplicacoes Ambientais. Embrapa.

Yamamotto, J., Landim, P., 2013. Geoestatística - Conceitos e Aplicações. OFICINA DE TEXTOS. 